\title{
Body Mass Index in Different Dementia Disorders: Results from the Swedish Dementia Quality Registry (SveDem)
}

\author{
Gerd Faxén-Irving a, c Seyed-Mohammad Fereshtehnejad ${ }^{a}$ \\ Farshad Falahati $^{a}$ Lars Cedergren ${ }^{a}$ Helen Göranzon ${ }^{d}$ Kristine Wallman ${ }^{d}$ \\ Sara García-Ptacek ${ }^{a}$ f Maria Eriksdotter ${ }^{a}$ b Dorota Religa ${ }^{a}$ b, e \\ a Department of Neurobiology, Care Sciences and Society Karolinska Institutet, \\ Departments of ${ }^{b}$ Geriatric Medicine and ${ }^{c}$ Clinical Nutrition and Dietetics, \\ Karolinska University Hospital, Stockholm, and ${ }^{d}$ Department of Food, Nutrition and

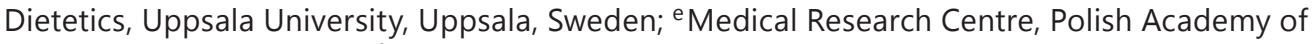 \\ Sciences, Warsaw, Poland; f Department of Medicine, Universidad Complutense de Madrid, \\ Madrid, Spain
}

\section{Key Words}

Dementia $\cdot$ Body mass index $\cdot$ Quality registry $\cdot$ Alzheimer's disease $\cdot$ Cognitive status

\begin{abstract}
Background: Most patients with dementia lose body weight over the course of the disease and have a lower body mass index (BMI) than subjects with normal cognition. Aims: To examine body mass index and how it correlates with cognitive status, age and gender in patients with different dementia disorders. Materials and Methods: Data from newly diagnosed dementia patients in the Swedish Dementia Quality Registry (SveDem) and recorded information about age, gender, cognitive status and BMI was analyzed using independent samples tests and one-way analysis of variance. Results: A total of 12,015 patients, 7,121 females and 4,894 males were included in the study. The average BMI was 24 . More than a quarter of the patients had a BMI of $<22$. Females were significantly older $(p<0.001)$ and males had a significantly higher BMI ( $p<0.001)$ at the time of diagnosis. BMI differed significantly by gender in various dementia disorders and correlated significantly with cognitive status and age. Conclusion: At the time of diagnosis, patients with various dementia disorders had a BMI within the normal range. However, a significant number had a BMI in a lower, suboptimal range for older persons stressing the need for nutritional assessment as part of the dementia work up. Further analyses with longitudinal follow-up are needed to investigate BMI changes over time.
\end{abstract}


Faxén-Irving et al:: Body Mass Index in Different Dementia Disorders: Results from the Swedish Dementia Quality Registry (SveDem)

\section{Introduction}

Dementia is a devastating disorder that burdens the patient, the family and society. It is expected that problems related to dementia will increase, especially in low-income and middleincome countries [1]. An increase in life expectancy contributes to the dementia epidemic. The number of older individuals is increasing particularly rapidly in China, India and Latin America, as are the proportions of older individuals in the population. It has been estimated that in 2050 close to $80 \%$ of subjects $>60$ years of age will live in the less developed regions in the world [1]. Further, it has been estimated that the number of dementia patients in the world will increase from the current value of 35,6-65,7 million by 2030 , and to 115,4 million by 2050 [2]. In Sweden, 20,000-25,000 subjects are diagnosed with dementia each year and about 150,000 individuals currently suffer from dementia. The annual cost of dementia disorders in Sweden is SEK 50 billion $[3,4]$. Approximately $1 \%$ of the population in Sweden suffers from a dementia disorder at 65 years of age and nearly $50 \%$ at 90 years of age [5].

\section{Overweight and Obesity}

It is well known that obesity increases the risk for vascular disorders such as atherosclerosis, chronic heart disease, hypertension and diabetes [6]. It has been suggested that these vascular disorders increase the probability that dementia will develop, particularly Alzheimer's disease (AD) and vascular dementia (VaD) [7, 8].

Body mass index (BMI) is a simple index of weight and height that is commonly used to classify overweight and obesity in adults [9]. BMI is defined as a person's weight in kg divided by the square of his or her height in meters $\left(\mathrm{kg} / \mathrm{m}^{2}\right)$. Individuals whose BMI is $>25$ are considered overweight [9]. Obesity is defined as having a BMI of $>30$, and the incidence of obesity is increasing at a high rate throughout the world. The prevalence of overweight in the USA and Europe is $>50 \%$. Epidemiological studies focusing on the relationship between BMI and the risk of developing dementia have consistently shown different results in mid- versus late-life. A high BMI late in life is associated with a low risk of dementia [10-12], while a high BMI in midlife is associated with a higher risk of dementia [12-14]. BMI provides a useful population-level measure of overweight and obesity, as it is the same for both sexes and for all adult ages. It may not, however, correspond to the same degree of fatness in different individuals [9]. The relationship between BMI and longevity has been extensively studied. Overweight subjects have a similar relative risk of mortality as subjects who are not overweight, while the risk of mortality for underweight subjects $(\mathrm{BMI}<18.5)$ and obese subjects (BMI $>30$ ) is higher [15]. Several studies indicate that the optimal range for BMI is increased for older subjects, and that higher BMI cutoff points should be used for elderly ( $<65$ years) [16-18].

\section{Weight Loss, Malnutrition and BMI in Dementia}

Weight loss and malnutrition are frequent in elderly individuals, and in individuals with dementia in particular [19]. Some studies have shown that patients with AD experience greater and more frequent weight loss compared with cognitively functioning elderly and subjects with $\operatorname{VaD}[19,20]$. Between 30 and $40 \%$ of patients with mild to moderate forms of $\mathrm{AD}$ experience a weight loss of $4 \%$ or more in a year [21]. As dementia progresses, severe weight loss becomes a serious problem, especially among the institutionalized elderly. Approximately $50 \%$ of institutionalized patients with dementia, especially $\mathrm{AD}$, suffer from protein-energy malnutrition [22]. With a probable diagnosis of AD, low BMI and weight loss are predictors of morbidity [23], mortality [24] and increased rates of disease progression. Nutritional considerations and weight loss are most studied in AD. However, patients with other diagnosis, especially Lewy body dementia (LBD) may be even more at risk for malnutrition due to eating and swallowing difficulties. 
Faxén-Irving et al.: Body Mass Index in Different Dementia Disorders: Results from the Swedish Dementia Quality Registry (SveDem)

\section{The Multifactorial Etiology of Weight Loss}

The etiology of weight loss in demented patients seems to be multifactorial. Potential contributing factors are: inability to prepare and eat foods [25], impairment of olfaction and taste, behavior problems like agitation, restlessness and wandering [26, 27], increased energy expenditure, inflammatory components and presence of comorbid medical illness.

The main objectives of this study were to characterize and compare BMI in patients with different dementia disorders at the time of diagnosis. In addition, we explored associations between BMI and cognitive status, age, and gender in different dementia disorders.

\section{Materials and Methods}

\section{Dataset}

Data was obtained from patients newly diagnosed with a dementia disorder, according to the ICD-10 classification, registered in the Swedish Dementia Quality Registry (SveDem; www.svedem.se) between May 1, 2007 and December 31, 2011.

The SveDem is a national quality registry which was started in 2007. Its purpose is to improve the quality of diagnostics, treatment and care of patients with dementia disorders $[3,28,29]$. The registry contains information such as age, gender, heredity, BMI, Mini-Mental State Examination (MMSE) scores, diagnoses, dementia work up investigations, medical treatment, and support from community and time from referral to diagnosis [3]. This information is registered for newly diagnosed patients and patients are followed up yearly. The Uppsala Clinical Trial Center, Uppsala, Sweden, is responsible for the online database and IT support. Today most information to the SveDem is provided by memory clinics but any health care unit is also eligible to participate. The following dementia diagnoses are registered in the SveDem: AD, late-onset AD (LOAD), early-onset AD (EOAD), VaD, mixed AD/VaD, LBD, Parkinson's disease with dementia (PDD), frontotemporal dementia (FTD), unspecified dementia (NOS) and other types of dementia disorders (including alcohol dementias and rare dementia disorders such as corticobasal degeneration).

\section{BMI Cutoff}

The optimal BMI in the elderly has not yet been defined. The European Society for Clinical Nutrition and Metabolism (ESPEN) and the national guidelines suggest that an older individual with a BMI of $<22$ should be considered as being at risk for malnutrition [30, 31]. In the present study, the following BMI cutoffs were chosen: $<22,22-25$ and $>25$. We also used the BMI cutoffs determined by the WHO for comparison. The WHO's cutoffs are $<18.5,18.5-22$, 22-25, 25-30 and $>30$.

\section{Statistics}

The Statistical Package for the Social Science (SPSS) software (IBM, USA) was used for statistical analysis. One-way analysis of variance (ANOVA) and independent samples tests were employed to explore associations between BMI and age at diagnosis, dementia diagnosis group and gender. For between-group comparisons, Bonferroni post hoc test was used. Bivariate correlations between pairs of continuous variables were also calculated. Analysis of covariance (ANCOVA) was applied to evaluate whether the mean differences in BMI between different types of dementia remained significant while statistically controlling for the effects of other continuous variables such as age. Multivariate linear regression analysis was used to compare the strength and independency of the relationship between MMSE score and several anthropometric measurements (BMI and weight) adjusted for patients' age and 
Dementia

and Geriatric

Table 1. Descriptive characteristics of the study population

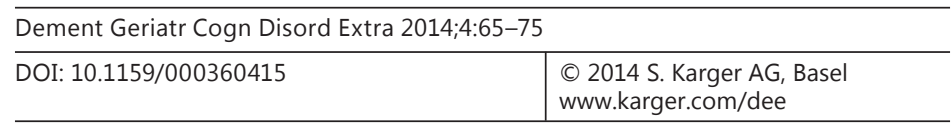

Faxén-Irving et al.: Body Mass Index in Different Dementia Disorders: Results from the Swedish Dementia Quality Registry (SveDem)

\begin{tabular}{llllc}
\hline & Total & Males & Females & p value \\
\hline Number & 12,015 & 4,894 & 7,121 & \\
Age, years & $78.6 \pm 7.9$ & $77.6 \pm 8.0$ & $79.3 \pm 7.8$ & $<0.001$ \\
BMI & $24.6 \pm 4.3$ & $25.1 \pm 3.9$ & $24.2 \pm 4.54$ & $<0.001$ \\
MMSE score & $21.4 \pm 5.0$ & $21.6 \pm 5.1$ & $21.2 \pm 5.0$ & 0.25 \\
\hline
\end{tabular}

Data are represented as means \pm standard deviation.

\begin{tabular}{lllll}
\hline BMI category & Patients, \% & Mean BMI & Age, years & MMSE score \\
\hline$<22$ & $28 \%$ & 19.9 & $80 \pm 8.0$ & $21 \pm 5.0^{*}$ \\
$22-25$ & $31 \%$ & 23.6 & $79 \pm 7.8$ & $22 \pm 5.0$ \\
$>25$ & $41 \%$ & 28.7 & $78 \pm 7.9$ & $22 \pm 5.0$
\end{tabular}

* Significant $(\mathrm{p}<0.05)$ compared to the other BMI groups.
Table 2. BMI divided into different BMI categories in all registered dementia patients

sex. All $\mathrm{p}$ values were based on two-sided tests and considered statistically significant if $<0.05$.

\section{Ethical Considerations}

Information, both orally and written is given to the patient before registration in the SveDem. Patients are given the opportunity to decline participation in the SveDem registry and can ask at any time to be removed from it. Ethical permission for this study has been obtained from the regional human ethics committee of Stockholm, Sweden.

\section{Results}

Between 2007 and 2011, 17,056 patients newly diagnosed with a dementia disorder were registered in the SveDem. Available BMI information obtained from 12,015 patients were included in the study. There were 7,121 females and 4,894 males distributed across nine different dementia diagnoses. The prevalence in percentage was: LOAD 32\%, mixed AD/ VaD 26\%, VaD 19\%, NOS 11\%, EOAD 4\%, LBD 3\%, FTD and other 2\%, and PDD 1\%.

Overall, 5,819 (49.0\%) of the patients were under treatment with cholinesterase inhibitors, and 1,193 (10.1\%) patients received NMDA antagonist (memantine) as anti-dementia medication. About $90 \%$ of the patients were living at home at the time of registration and were classified with mild dementia. Almost half of the patients had coresident(s)/caregiver(s) $(\mathrm{n}=6,048,51.7 \%)$. The average age at diagnosis was $79.3 \pm 7.8$ years for females and $77.6 \pm$ 8.0 years for males $(\mathrm{p}<0.001)$. Females had a lower BMI $(24.2)$ compared to males $(25.1$, $\mathrm{p}<0.001$ ) (table 1).

Seven of 10 patients were normal or overweight at the time of diagnosis. However, $28 \%$ of the patients were scored as underweight according to the $\mathrm{BMI}<22$ categorization (table 2 ). MMSE was significantly lower $(21 \pm 5)$ in patients with a BMI of $<22(\mathrm{p}<0.01)$. Using the BMI cutoff for $<18.5$ determined by the WHO for comparison, the significance between low BMI and cognitive impairment was even stronger $(p<0.001)$. 
Faxén-Irving et al.: Body Mass Index in Different Dementia Disorders: Results from the Swedish Dementia Quality Registry (SveDem)

Table 3. BMI in different dementia diagnoses

\begin{tabular}{llllr}
\hline Diagnosis & Total & Males & Females & p value $^{\mathrm{b}}$ \\
\hline EOAD & $24.37 \pm 4.1(486)$ & $24.70 \pm 3.5(167)$ & $24.20 \pm 4.4(319)$ & 0.169 \\
LOAD & $24.07 \pm 4.0(3,815)$ & $24.47 \pm 3.5(1,276)$ & $23.87 \pm 4.2(2,539)$ & $<0.001^{\mathrm{c}}$ \\
Mixed AD/VaD & $24.43 \pm 4.2(3,159)$ & $24.90 \pm 3.7(1,293)$ & $24.10 \pm 3.5(1,866)$ & $<0.001$ \\
VaD & $25.34 \pm 4.5(2,249)$ & $25.75 \pm 4.1(1,128)$ & $24.93 \pm 4.9(1,121)$ & $<0.001$ \\
LBD & $24.31 \pm 4.1(337)$ & $24.59 \pm 3.5(205)$ & $23.88 \pm 4.9(132)$ & 0.155 \\
FTD & $25.83 \pm 4.9(260)$ & $26.59 \pm 4.5(118)$ & $25.20 \pm 5.2(142)$ & $<0.020$ \\
PDD & $24.10 \pm 3.7(174)$ & $24.42 \pm 3.4(99)$ & $23.68 \pm 4.0(75)$ & 0.190 \\
NOS & $24.93 \pm 4.9(1,284)$ & $25.78 \pm 4.4(491)$ & $24.40 \pm 5.0(793)$ & $<0.001$ \\
Other & $24.39 \pm 4.5(251)$ & $24.72 \pm 4.4(117)$ & $24.09 \pm 4.6(134)$ & 0.269 \\
p value & & $<0.001$ & $<0.001$ & \\
\hline
\end{tabular}

Data are represented as means \pm standard deviation with numbers in parentheses.

${ }^{a}$ p value for ANOVA. ${ }^{b}$ p value for the independent sample $t$ test between males and females. ${ }^{\mathrm{c}}$ The lowest BMI was observed among patients diagnosed with LOAD $(\mathrm{p}<0.001)$.

Table 4. Dementia diagnoses categorized into different BMI groups

\begin{tabular}{lrrrr}
\hline & Total, $\mathrm{n}$ & $<22$ & $22-25$ & \multicolumn{1}{l}{$>25$} \\
\hline EOAD & 487 & $141(29.0)$ & $157(32.2)$ & $189(38.8)$ \\
LOAD & 3,810 & $1,189(31.2)$ & $1,233(32.4)$ & $1,388(36.4)$ \\
Mixed AD/VaD & 3,153 & $914(29.0)$ & $977(31.0)$ & $1,262(40.0)$ \\
VaD & 2,243 & $508(22.6)$ & $654(29.2)$ & $1,081(48.2)$ \\
LBD & 337 & $106(31.5)$ & $110(32.6)$ & $121(35.9)$ \\
FTD & 260 & $54(20.8)$ & $71(27.3)$ & $135(51.9)$ \\
PDD & 173 & $46(26.6)$ & $62(35.8)$ & $65(37.6)$ \\
NOS & 1,282 & $363(28.3)$ & $348(27.1)$ & $571(44.5)$ \\
Other & 250 & $76(30.4)$ & $75(30.0)$ & $99(39.6)$ \\
\hline
\end{tabular}

Values in parentheses are percentages.

\section{BMI by Type of Dementia}

BMI was analyzed separately by gender in each diagnosis group (table 3). Independent samples t test showed that in LOAD, mixed AD/VaD, VaD, FTD and NOS a significantly higher BMI was found for males compared to females. Moreover, one-way ANOVA analysis revealed that there were significant differences in BMI between diagnoses. The highest BMI was observed among patients diagnosed with FTD and VaD, while the lowest BMI was observed among patients diagnosed with LOAD $(\mathrm{p}<0.001)$. ANCOVA analysis showed that not only the type of dementia (LOAD vs. FTD; $F=18.2, p<0.001)$ but also the patients' age $(F=41.9, p<$ 0.001 ) were statistically significant factors for the difference observed in the mean BMI between the LOAD and FTD groups. BMI was also divided according to different BMI cutoffs (i.e. a BMI of $<22,22-25$, and $>25$ ) for each dementia diagnosis, showing no statistical differences between the groups in the percentage of patients with a BMI of $<22$ (table 4).

However, in FTD and VaD, the percentage of patients with a BMI of $<22$ was lower than for the other dementia groups, while the LBD and AD groups had the highest percentage of patients at risk for malnutrition (table 4).

\section{BMI and Cognitive Status}

The mean MMSE score was $21.4 \pm 5.0$. Bivariate correlation analysis showed weak but significant positive correlations between BMI and MMSE score (correlation coefficient = 
Faxén-Irving et al.: Body Mass Index in Different Dementia Disorders: Results from the Swedish Dementia Quality Registry (SveDem)

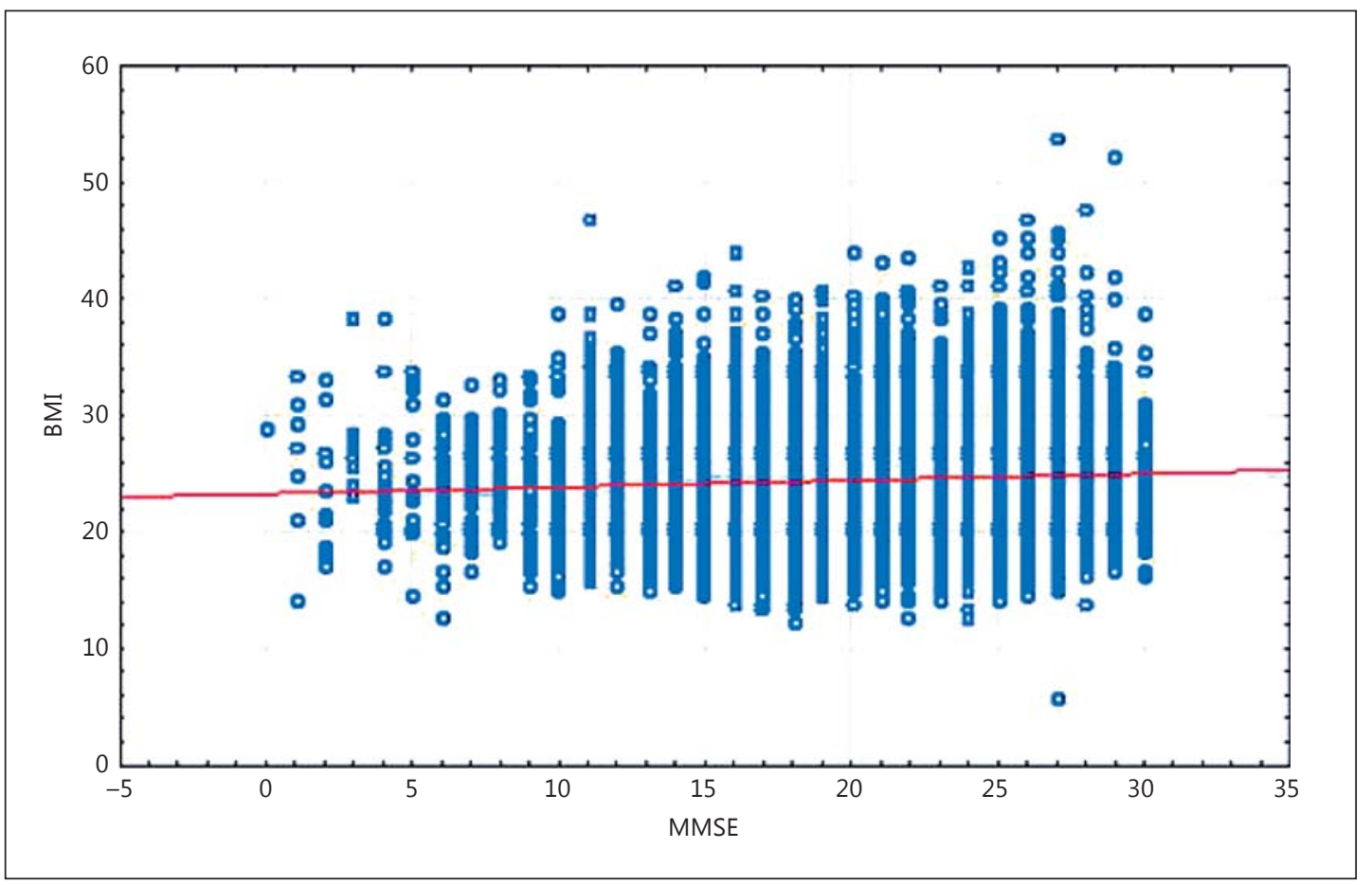

Fig. 1. Relationship between BMI and MMSE in all registered dementia patients. Correlation coefficient = $0.065, \mathrm{p}<0.001$.

0.065, $\mathrm{p}<0.001$ ) (fig. 1). The weak positive correlation between BMI and MMSE score remained significant ( $B=0.052,95 \%$ CI 0.031-0.074) even after adjustment for type of dementia, gender and age at the time of diagnosis (table 5). The correlation was negative (inverse) between BMI and age (correlation coefficient $=-0.127, \mathrm{p}<0.001$ ).

Figure 2 shows the proportion of patients (\%) in the different MMSE groups and the different BMI categories. The highest proportion of patients with a MMSE score of $<9(3.2 \%)$ and a MMSE score of 10-20 (46.7\%) was found in patients with a BMI of $<18.5$; these prevalence rates gradually decreased with increasing BMI.

\section{Discussion}

The present study shows that the prevalences of different diagnoses in SveDem are in accordance with those reported in the literature. Men had significantly higher BMI and women were significantly older, as has been shown in the general population. At the time of diagnosis, the majority of patients had a mild stage of dementia and a normal BMI, which is in accordance with other studies [32,33]. The mean BMI in VaD and FTD patients was significantly higher as compared to BMI in other dementia disorders. This difference could be caused by the behaviour changes in FTD, where hyperphagia, characterized by eating excessive amounts of food is a common symptom. For VaD it is known that overweight and obesity increase the risk for vascular events, which could explain the higher BMI in this group. With age, BMI decreases and this may be considered as a pathological process or as a part of the normal aging process. The LOAD group had the lowest BMI, which could be due to the fact that LOAD is diagnosed later in life compared to other dementia diagnoses; therefore, BMI is lower at 
Faxén-Irving et al.: Body Mass Index in Different Dementia Disorders: Results from the Swedish Dementia Quality Registry (SveDem)

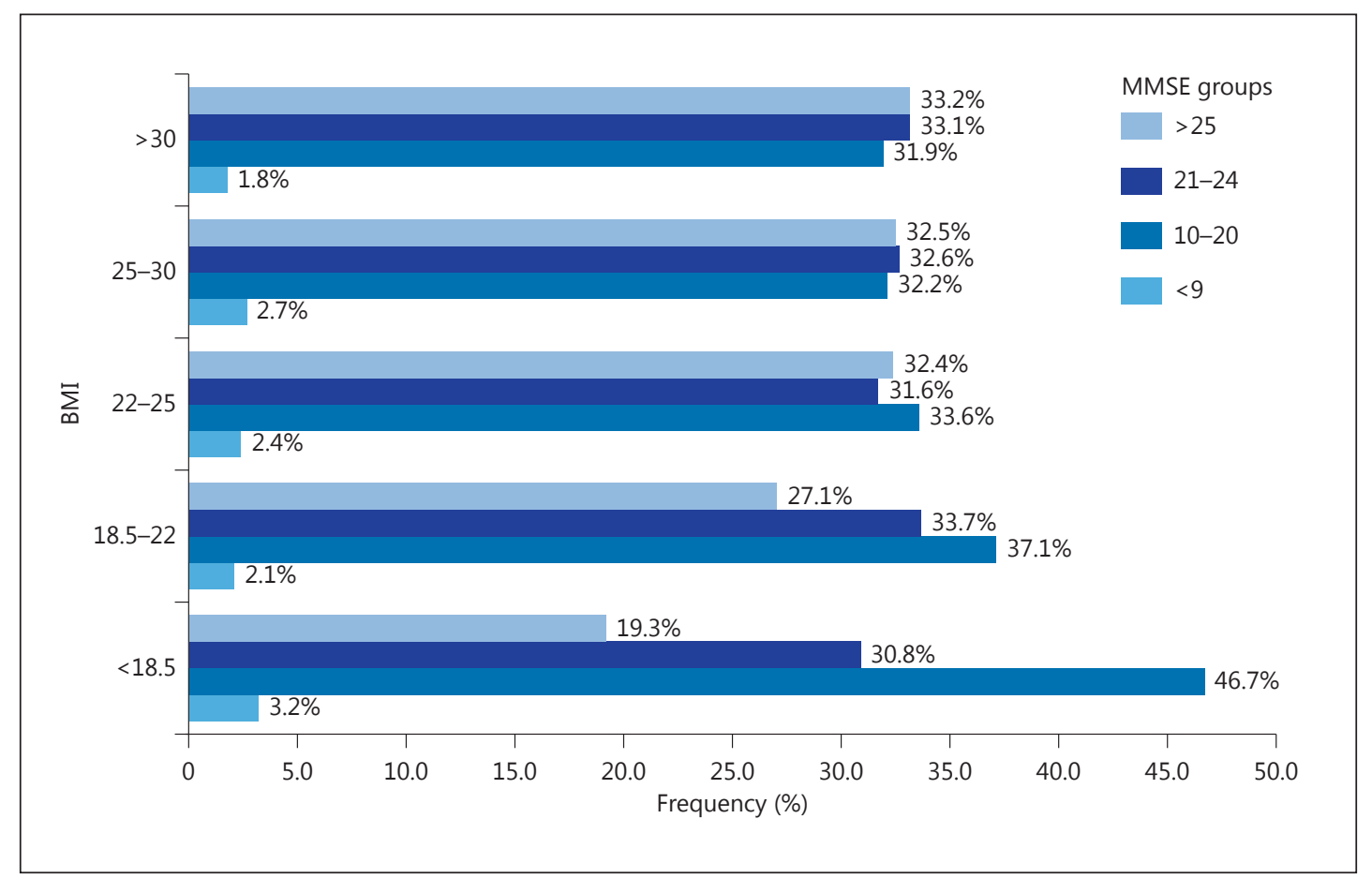

Fig. 2. Frequency of different levels of cognitive impairment (based on the MMSE score) in the different categories of BMI.

Table 5. Multivariate linear regression model to evaluate the effects of BMI and some confounder variables on the MMSE score in registered dementia patients

\begin{tabular}{|c|c|c|c|c|c|c|c|}
\hline & \multirow[t]{2}{*}{ B } & \multirow[t]{2}{*}{ SE } & \multicolumn{2}{|c|}{$\begin{array}{l}95 \% \text { Wald confidence } \\
\text { interval }\end{array}$} & \multicolumn{3}{|c|}{ Hypothesis test } \\
\hline & & & lower & upper & Wald $\chi^{2}$ & d.f. & $\mathrm{p}$ value \\
\hline Intercept & 27.413 & 0.6833 & 26.074 & 28.752 & $1,609.592$ & 1 & $<0.001$ \\
\hline Male gender & 0.121 & 0.0959 & -0.067 & 0.309 & 1.601 & 1 & 0.206 \\
\hline \multicolumn{8}{|l|}{ Type of dementia } \\
\hline EOAD & -0.145 & 0.4097 & -0.948 & 0.658 & 0.126 & 1 & 0.723 \\
\hline LOAD & 0.869 & 0.3439 & 0.195 & 1.543 & 6.382 & 1 & 0.012 \\
\hline Mixed AD/VaD & 0.546 & 0.3474 & -0.135 & 1.227 & 2.470 & 1 & 0.116 \\
\hline $\mathrm{VaD}$ & 0.768 & 0.3515 & 0.079 & 1.457 & 4.776 & 1 & 0.029 \\
\hline LBD & 0.632 & 0.4311 & -0.213 & 1.476 & 2.146 & 1 & 0.143 \\
\hline FTD & 1.993 & 0.4614 & 1.088 & 2.897 & 18.653 & 1 & $<0.001$ \\
\hline PDD & 0.004 & 0.5103 & -0.996 & 1.004 & 0.000 & 1 & 0.993 \\
\hline NOS & -0.493 & 0.3631 & -1.205 & 0.219 & 1.845 & 1 & 0.174 \\
\hline Other & ref. & - & - & - & - & - & - \\
\hline Age & -0.101 & 0.0067 & -0.114 & -0.088 & 224.745 & 1 & $<0.001$ \\
\hline BMI & 0.052 & 0.0109 & 0.031 & 0.074 & 22.901 & 1 & $<0.001$ \\
\hline
\end{tabular}


Faxén-Irving et al.: Body Mass Index in Different Dementia Disorders: Results from the Swedish Dementia Quality Registry (SveDem)

the time of diagnosis. The patients in the present study were significantly older than the FTD patients. LOAD patients, also suffer from age-related factors such as frailty, sarcopenia, functional impairments and comorbidities besides their disease-related problems (behavior problems, inflammation and olfactory deficits) which all may result in weight loss and low BMI in contrast to patients with FTD $[25,27]$. The patients with suboptimal BMI $(<22 ; 28 \%)$ also had a significantly lower cognitive function than those with higher BMI. There was a positive but weak relationship between BMI and MMSE. The cross-sectional design of our study does not allow us to draw conclusions regarding causality.

According to the ESPEN and the Swedish national recommendations [30, 31], underweight $(\mathrm{BMI}<22)$ is a risk factor for malnutrition in older subjects. Most nutritional screening tools include BMI but also information about weight change, appetite and questions related to eating. The Mini-Nutritional Assessment (MNA) was developed for use in a geriatric population to identify persons at nutritional risk [34]. The MNA has been used in both non-demented and demented populations. The MNA SF is a short and less time-consuming version [35].

The fact that almost one third of the 12,015 patients had a suboptimal BMI suggestive of a risk for malnutrition should stress the need for nutritional assessment and intervention. Practical guidelines for the diagnosis and management of malnutrition in AD suggest a nutritional assessment at the time of diagnosis and in the follow-up and management of the disease [36]. Patients with a BMI of $<22$ were more cognitively impaired compared to normal and overweight patients. When using a BMI cutoff of $<18.5$, the significance association between low BMI and cognitive impairment becomes even stronger. This significant difference in cognitive function among those classified as underweight could be due to the fact that the diagnosis was established in a later stage of the disease and thus weight loss may already have started $[32,33]$. Other plausible factors are malnutrition, causing or caused by cognitive decline, or other chronic diseases resulting in underweight. Involuntary weight loss should always be examined for any malignancy or food intake-related impairments.

There is yet not sufficient evidence on efficacy interventions aimed to avoid and treat dementia-related weight loss. However, some systematic reviews on non-pharmacological interventions such as nutritional support [37-39], progressive resistive exercise and aerobic exercise, report weight gain and increased oral intake effects. Positive effects by oral supplements are confirmed in a systematic review [40]. Furthermore, multidomain interventions suggest that strategies which target multiple factors simultaneously may prove more effective than focusing on a single mechanism or domain [41].

\section{Strengths and Limitations}

The primary strength of this study is the large population number included in the SveDem. The SveDem is one of the largest dementia registries in the world covering $30 \%$ of newly diagnosed patients in Sweden. The patients are diagnosed in different parts of the country, reflecting real medical practice, without many inclusion or exclusion criteria. One limitation is that the number of missing BMI data (usually length) is quite high (30\%). The benefits with registries are large cohorts, but the risk that registration is not performed properly is always present, leaving large numbers of missing data. To ensure good registry quality it is necessary to improve the registration process in order to minimize the amount of missing data. The SveDem is already monitoring units by validating random patient registrations against the medical records.

The cross-sectional design of our study is another limitation from the point of view of discussing causality. We do not yet have enough information to study BMI changes over time. It would also be of interest to study BMI in this population 5-10 years before the diagnosis of dementia, which may be possible combining data from other registries. Furthermore, the SveDem provides no information on the nutritional status, socioeconomic variables, physical activities or the patients' diet. 


\section{Clinical Implication}

The mean BMI was similar in all dementia disorders studied, and although some statistical differences were found these are of minor clinical importance. However, clinically relevant is that almost $30 \%$ of the patients were underweight with a BMI of $<22$ already at the time of diagnosis. Thus, there is a need to implement a routine for nutritional screening of all patients at the time of diagnosis to find patients at risk for malnutrition. Practical guidelines for the diagnosis and management of malnutrition in AD have suggested a nutritional assessment at the time of diagnosis and at follow-up [36]. Most studies conducted on dementia and nutrition include predominantly AD patients. This study includes data on BMI also from other dementia disorders. Without denying the fact that a different dementia diagnosis may have different biological mechanisms for weight loss, intervention may be designed on the basis of current evidence-based knowledge and clinical experiences.

\section{Future Studies}

As all registry-based studies, this study aims to inspire new hypotheses to be researched in the future. The epidemiological data presented here can be used as basis for intervention studies to prevent weight loss during the course of dementia. Future studies should focus on long-term follow-up. An intervention study on the group with a BMI of $<22$ would also be of interest.

\section{Conclusion}

The mean BMI in patients registered in the SveDem did not differ between different dementia disorders but was slightly different between men and women. The majority of patients newly diagnosed with dementia had a normal BMI, but almost a third was underweight having a BMI of $<22$. Therefore, it is important to perform nutritional screening and assessment of all patients with dementia at the time of dementia diagnosis, as well as to follow BMI changes over time. Data from the SveDem can contribute to the implementation of such a routine.

\section{Acknowledgements}

The authors are grateful to the national Swedish Dementia Quality Registry (SveDem; www.svedem.se) for providing data for this study and thank all patients, caregivers, and reporting units for providing information.

This study was supported by grants from the Swedish Society of Medicine, Karolinska Institutets Foundation, the Foundation for Geriatric Diseases at Karolinska Institutet, the Swedish Dementia Foundation, Swedish Alzheimer and Dementia Research Foundation, Stiftelsen for Gamla Tjänarinnor, the Swedish Brain Power Network and the Swedish Association of Local Authorities and Regions.

\section{Disclosure Statement}

The authors have neither conflicts of interest nor any affiliations with the industry related to this work. 
Faxén-Irving et al.: Body Mass Index in Different Dementia Disorders: Results from the Swedish Dementia Quality Registry (SveDem)

\section{References}

1 Prince M, Bryce R, Albanese E, Wimo A, Ribeiro W, Ferri CP: The global prevalence of dementia: a systematic review and metaanalysis. Alzheimers Dement 2013;9:63-75.e2.

-2 Wimo A, Jonsson L, Bond J, Prince M, Winblad B; Alzheimer Disease International. The worldwide economic impact of dementia 2010. Alzheimers Dement 2013;9:1-11.e3.

-3 Religa D, Spangberg K, Wimo A, Edlund AK, Winblad B, Eriksdotter-Jonhagen M: Dementia diagnosis differs in men and women and depends on age and dementia severity: data from SveDem, the Swedish Dementia Quality Registry. Dement Geriatr Cogn Disord 2012;33:90-95.

-4 Wimo A, Jonsson L, Gustavsson A, McDaid D, Ersek K, Georges J, Gulácsi L, Karpati K, Kenigsberg P, Valtonen $\mathrm{H}$ : The economic impact of dementia in Europe in 2008-cost estimates from the Eurocode project. Int J Geriatr Psychiatry 2011;26:825-832.

5 SBU: Dementia - etiology and epidemiology. a systematic review. The Swedish Council on Technology Assessment in Health Care, 2008, Contract No.: No. 172E/1.

6 Kopelman PG: Obesity as a medical problem. Nature 2000;404:635-643.

7 Skoog I, Kalaria RN, Breteler MM: Vascular factors and Alzheimer disease. Alzheimer Dis Assoc Disord 1999; 13(suppl 3):S106-S114.

-8 Hofman A, Ott A, Breteler MM, Bots ML, Slooter AJ, van Harskamp F, van Duijn CN, Van Broeckhoven C, Grobbee DE: Atherosclerosis, apolipoprotein E, and prevalence of dementia and Alzheimer's disease in the Rotterdam Study. Lancet 1997;349:151-154.

9 WHO Technical Report Series. Obesity: preventing and managing the global epidemic. WHO, 2000.

10 Buchman AS, Wilson RS, Bienias JL, Shah RC, Evans DA, Bennett DA: Change in body mass index and risk of incident Alzheimer disease. Neurology 2005;65:892-897.

-11 Gustafson DR, Backman K, Waern M, Ostling S, Guo X, Zandi P, Mielke MM, Bengtsson C, Skoog I: Adiposity indicators and dementia over 32 years in Sweden. Neurology 2009;73:1559-1566.

-12 Kivipelto M, Ngandu T, Fratiglioni L, Viitanen M, Kareholt I, Winblad B, Helkala EL, Tuomilehto J, Soininen H, Nissinen A: Obesity and vascular risk factors at midlife and the risk of dementia and Alzheimer disease. Arch Neurol 2005;62:1556-1560.

13 Whitmer RA, Gustafson DR, Barrett-Connor E, Haan MN, Gunderson EP, Yaffe K: Central obesity and increased risk of dementia more than three decades later. Neurology 2008;71:1057-1064.

14 Whitmer RA, Gunderson EP, Quesenberry CP Jr, Zhou J, Yaffe K: Body mass index in midlife and risk of Alzheimer disease and vascular dementia. Curr Alzheimer Res 2007;4:103-109.

15 Flegal KM, Graubard BI, Williamson DF, Gail MH: Excess deaths associated with underweight, overweight, and obesity. JAMA 2005;293:1861-1867.

16 Beck AM, Ovesen L: At which body mass index and degree of weight loss should hospitalized elderly patients be considered at nutritional risk? Clin Nutr 1998;17:195-198.

$\checkmark 17$ Flodin L, Svensson S, Cederholm T: Body mass index as a predictor of 1 year mortality in geriatric patients. Clin Nutr 2000;19:121-125.

18 Flicker L, McCaul KA, Hankey GJ, Jamrozik K, Brown WJ, Byles JE, Almeida OP: Body mass index and survival in men and women aged 70 to 75. J Am Geriatr Soc 2010;58:234-241.

19 White H, Pieper C, Schmader K, Fillenbaum G: Weight change in Alzheimer's disease. J Am Geriatr Soc 1996; 44:265-272.

20 Gillette-Guyonnet S, Nourhashémi F, Andrieu S, de Glisezinski I, Ousset JP, Rivière D, Albarède J-L, Vellas B: Weight loss in Alzheimer's disease. Am J Clin Nutr 2000;71:637S-642S.

21 Gillette Guyonnet S, Abellan Van Kan G, Alix E, Andrieu S, Salva A: IANA (International Academy on Nutrition and Aging) Expert group: weight loss and Alzheimer's disease. J Nutr Health Aging 2007;11:38-48.

-22 Guigoz Y, Lauque S, Vellas BJ: Identifying the elderly at risk for malnutrition. The Mini Nutritional Assessment. Clin Geriatr Med 2002;18:737-757.

23 Mazzali G, Bissoli L, Gambina S, Residori L, Pagliari P, Guariento S, Sun M, Broggio E, Bosello O, Zamboni M: Energy balance in Alzheimer's disease. J Nutr Health Aging 2002;6:247-253.

24 White H, Pieper C, Schmader K: The association of weight change in Alzheimer's disease with severity of disease and mortality: a longitudinal analysis. J Am Geriatr Soc 1998;46:1223-1227.

-25 Fjellström C, Starkenberg A, Wesslén A, Tysén Bäckström AC, Faxén-Irving G; OmegAD Study Group: To be a good food provider: an exploratory study among spouses of persons with Alzheimer's disease. Am J Alzheimers Dis Other Demen 2010;25:521-526.

26 Young KW, Greenwood CE: Shift in diurnal feeding patterns in nursing home residents with Alzheimer's disease. J Gerontol A Biol Sci Med Sci 2001;56:700-706.

-27 White HK, McConnell ES, Bales CW, Kuchibhatla M: A 6-month observational study of the relationship between weight loss and behavioral symptoms in institutionalized Alzheimer's disease subjects. J Am Med Dir Assoc 2004;5:89-97.

28 Johnell K, Religa D, Eriksdotter M: Differences in drug therapy between dementia disorders in the Swedish dementia registry: a nationwide study of over 7,000 patients. Dement Geriatr Cogn Disord 2013;35:239-248.

29 Wimo A, Religa D, Spangberg K, Edlund AK, Winblad B, Eriksdotter M: Costs of diagnosing dementia: results from SveDem, the Swedish Dementia Registry. Int J Geriatr Psychiatry 2013;10:1039-1044. 
30 Sobotka L, Schneider SM, Berner YN, Cederholm T, Krznaric Z, Shenkin A, Stanga Z, Toigo G, Vandewoude M, Volkert D: ESPEN Guidelines on Parenteral Nutrition: geriatrics. Clin Nutr 2009;28:461-466.

31 Socialstyrelsen. Näring för god vård och omsorg - en vägledning för att förebygga och behandla undernäring; in: Socialstyrelsen, editor. www.socialstyrelsen.se/publikationer2011/2011-9-22011.

-32 Power BD, Alfonso H, Flicker L, Hankey GJ, Yeap BB, Almeida OP: Changes in body mass in later life and incident dementia. Int Psychogeriatr 2013;25:467-478.

33 Coin A, Veronese N, De Rui M, Mosele M, Bolzetta F, Girardi A, Manzato E, Sergi G: Nutritional predictors of cognitive impairment severity in demented elderly patients: the key role of BMI. J Nutr Health Aging 2012;16: 553-556.

34 Guigoz Y, Vellas B, Garry PJ: Mini Nutritional Assessment: a practical assessment tool for grading the nutritional state of elderly patients. Facts Res Geront 1994;2:15-59.

35 Kaiser MJ, Bauer JM, Ramsch C, Uter W, Guigoz Y, Cederholm T, Thomas DR, Anthony P, Charlton KE, Maggio M, Tsai AC, Grathwohl D, Vellas B, Sieber CC; MNA-International Group: Validation of the Mini Nutritional Assessment short-form (MNA-SF): a practical tool for identification of nutritional status. J Nutr Health Aging 2009;13:782-788.

-36 Belmin J; Expert Panel and Organisation Committee: Practical guidelines for the diagnosis and management of weight loss in Alzheimer's disease: a consensus from appropriateness ratings of large expert panel. J Nutr Health Aging 2007;11:33-37.

37 Payne C, Wiffen PJ, Martin S: Interventions for fatigue and weight loss in adults with advanced progressive illness. Cochrane Database Syst Rev 2012;1:CD008427.

38 Hanson LC, Ersek M, Gilliam R, Carey TS: Oral feeding options for people with dementia: a systematic review. J Am Geriatr Soc 2011;59:463-472.

-39 Liu W, Cheon J, Thomas SA: Interventions on mealtime difficulties in older adults with dementia: a systematic review. Int J Nurs Stud 2013, Epub ahead of print.

40 Allen VJ, Methven L, Gosney MA: Use of nutritional complete supplements in older adults with dementia: systematic review and meta-analysis of clinical outcomes. Clin Nutr 2013, Epub ahead of print.

41 Schneider N, Yvon CJ: A review of multidomain interventions to support healthy cognitive ageing. Nutr Health Aging 2013;17:252-257. 